\title{
Spider silk: a novel optical fibre for biochemical sensing
}

\author{
Kenny Hey Tow ${ }^{a}$, Desmond M. Chow ${ }^{a}$, Fritz Vollrath ${ }^{b}$, Isabelle Dicaire ${ }^{c}$, Tom Gheysens ${ }^{c}$ and \\ Luc Thévenaz ${ }^{a}$ \\ ${ }^{a}$ EPFL Swiss Federal Institute of Technology, Group for Fibre Optics, SCI-STI-LT, Station \\ 11, CH-1015 Lausanne, Switzerland; \\ ${ }^{b}$ University of Oxford, Department of Zoology, Oxford OX1 3PS, UK; \\ ${ }^{c}$ Advanced Concepts Team, European Space Agency (ESA), Noordwijk, The Netherlands.
}

\begin{abstract}
Whilst being thoroughly used in the textile industry and biomedical sector, silk has not yet been exploited for fibre optics-based sensing although silk fibres directly obtained from spiders can guide light and have shown early promises to being sensitive to some solvents. In this communication, a pioneering optical fibre sensor based on spider silk is reported, demonstrating for the first time the use of spider silk as an optical fibre sensor to detect polar solvents such as water, ammonia and acetic acid.
\end{abstract}

Keywords: Biosensing, optical fibre sensor, spider silk, birefringence

\section{INTRODUCTION}

In the past few years, optical sensors have been under intensive investigation and development as biological and chemical sensors. Small size, high sensitivity, high selectivity and low detection limits are the dominant requirements for these applications. Promising alternatives to conventional biosensors are fibre-optic biosensors (FOBs), which are optical fibre-based devices used to measure biological molecular species ${ }^{1}$ such as proteins, nucleic acids, etc. These encompass all the advantages linked to the use of optical fibres: low cost, small size, chemical-inertness, compatibility to a wide range of surface modifications, the potential for remote sensing, with the use and availability of inexpensive lasers and photodetectors.

(a)

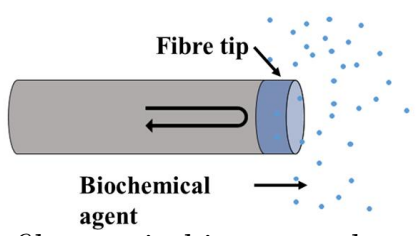

(b)

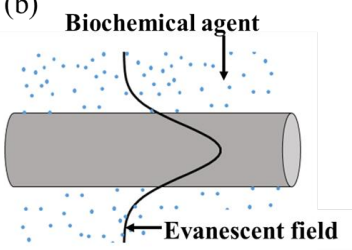

(c)

B) Biochemical agent

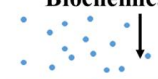

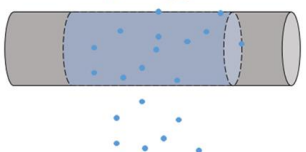

Figure 1: Typical fibre-optic biosensors based on (a) fibre tip, (b) evanescent field sensing and (c) proposed simplified architecture in this communication.

The working principle of typical FOBs requires direct interaction between light and the agent to be detected. In these systems, the optical fibre's sole purpose is to transport light to and from the sensing region whether the operating mechanism is based on fibre tip technology (Figure 1(a)) or evanescent field sensing (Figure 1(b)). A more innovative and straightforward approach is to directly use the optical fibre itself as the sensing element, i.e. the chemical or biological agent modifies the properties of the fibre material in its entire volume (Figure 1(c)). The general principle of such a sensor is outlined as follows: light from an optical source is sent through the sensing fibre, the agent will interact with the fibre material and any induced change in the fibre's properties due to the presence of the agent will subtly alter the parameters of the transmitted light (intensity, phase, spectrum, polarisation), which can be analysed and interpreted subsequently. In this way, the architecture of the FOB would be considerably simplified and less energy-consuming compared to classical FOBs.

Further author information: send correspondence to Kenny Hey Tow (kenny.heytow@epfl.ch)

24th International Conference on Optical Fibre Sensors, edited by Hypolito José Kalinowski, José Luís Fabris, Wojtek J. Bock, Proc. of SPIE Vol. 9634, 96347D · @ 2015 SPIE CCC code: $0277-786 \mathrm{X} / 15 / \$ 18 \cdot$ doi: $10.1117 / 12.2192427$ 
Silica optical fibres are commonly used for sensing physical parameters such as temperature, mechanical strain, vibrations, pressure acceleration, rotations (gyroscopes) but they are very poorly or even not at all sensitive to chemical or biological compounds, restricting the implementations to tip or evanescent field sensing. ${ }^{2}$ Considering the high solidification temperature of silica, complex reactive molecules cannot be incorporated in the silica material while keeping their chemical integrity. Hence, the motivation behind this work is to use an optical fibre made of a material whose mechanical and optical properties would be potentially altered in presence of biochemical agents. The perfect candidate in this case is spider silk since its properties can be consistently tuned as necessary for detecting any type of biochemical agent (gasses, solvents etc.) in a very cost-effective way as it is spun at ambient conditions. In contrast to natural fibres such as wool, which are generally cross-linked, silk fibres are held together by reversible hydrogen bonding that can be changed by polar solvents making them ideal for detecting modifying

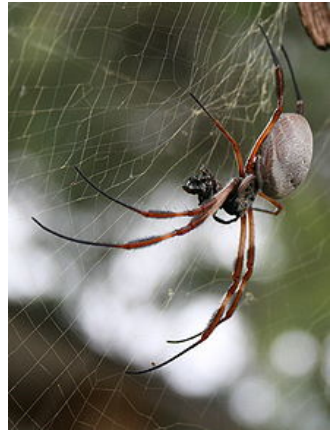

Figure 2: A female Nephila edulis spider agents such as water molecules, acids, bases, etc. Earlier research has shown that spider silk supercontracts in water as well as in a range of polar solvents such as methanol, ethanol and urea solutions. ${ }^{3}$ Very recently light guiding in spider dragline silk has been demonstrated in the region of $800 \mathrm{~nm},{ }^{4}$ though with very high losses, thereby paving the way towards the use of silk fibres for biochemical sensing.

\section{OPTICAL CHARACTERISATION OF THE SPIDER SILK FIBRE}

A single major ampullate silk fibre from a female Nephila edulis spider (Figure 2) was reeled onto a spool by the Oxford Silk Group. The reeling process under controlled speed ${ }^{5}$ resulted in very uniformly spun fibres with a smooth surface, equal circular diameters and identical material properties, which is of vital importance for its optical properties. Silk fibres with a diameter of $5.6 \mu \mathrm{m}$ were collected for our experiments. When surrounded by air, the silk, with a reported refractive index $n$ of around 1.55 in the visible spectrum, ${ }^{6}$ is intrinsically an optical fibre and guides light by total internal reflection with refractive index contrast $\Delta n$ of 0.55 .

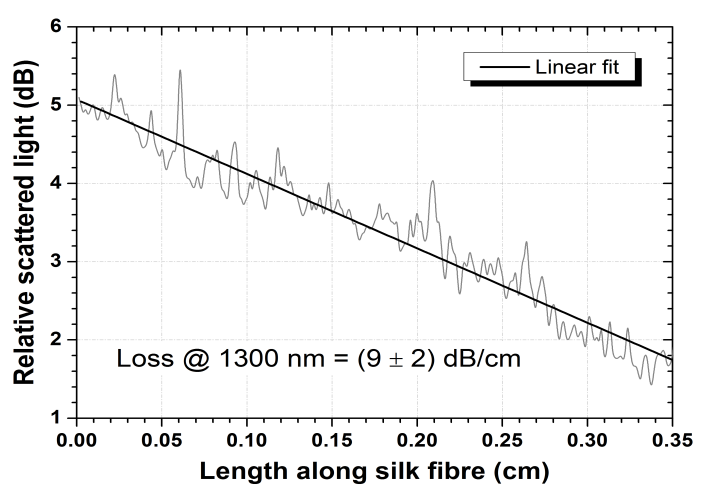

(a)

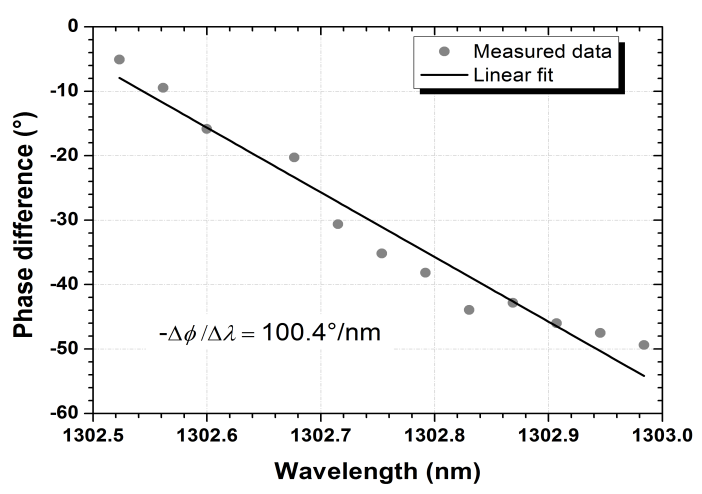

(b)

Figure 3: (a) Intensity of scattered light measured along the silk fibre and (b) phase difference as a function of wavelength.

To prepare a dragline silk sample for light injection, the silk fibre was kept tensioned and fixed on a dedicated holder using optical grade glue with refractive index $n$ of 1.46. Two standard single mode fibres (SMF-28) were placed on multi-axis precision stages to inject and collect light from the silk fibre. Challenges arose when handling the spider silk due to its microscopic size and wobbly nature. Therefore, they were kept under a small tension force for light injection thus prohibiting cleaving or breaking as in conventional silica-based optical fibre handling. This complicated the use of cut-back method to estimate the attenuation coefficient $\alpha$, prompting the use of a non-invasive method, namely the scattered light image analysis technique, which is commonly used for determining optical losses in optical waveguides. ${ }^{7}$ A top view detection system to observe the scattered light from the silk fibre was configured with a charge coupled device (CCD) camera sensitive in both visible spectrum and near infrared region. The output video signal was compiled into a light intensity distribution map. The peak intensity variation along a section of the silk fibre was acquired along the propagation direction and $\alpha$ was 
calculated from the longitudinal change as indicated in Figure 3(a). Measurements were made on several 0.35 $\mathrm{cm}$ segments from two different silk fibre samples from the same dragline spool. After averaging, propagation loss is estimated as $2 \pm 1 \mathrm{~dB} / \mathrm{cm}$ for red light and $9 \pm 2 \mathrm{~dB} / \mathrm{cm}$ in the O-band region.

The birefringence of the silk fibre at $1302 \mathrm{~nm}$ was also experimentally evaluated using a wavelength-scanning method. ${ }^{8}$ Light with a circular polarization was injected into a silk fibre of length $L=2.5 \mathrm{~cm}$ and the wavelength dependence of the polarization phase difference $\phi$ induced by the silk fibre was measured at the output using a polarization analyser and plotted on Figure 3(b). From this graph, a value of 0.0189 was calculated for the birefringence $B$ of the silk fibre using the following equation: $B=-\frac{\Delta \phi}{\Delta \lambda} \cdot \frac{\lambda^{2}}{2 \pi L}$.

\section{DETECTION OF BIOCHEMICAL AGENTS WITH SILK FIBRE}

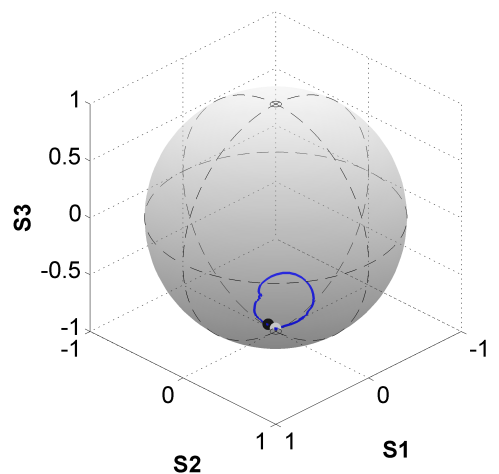

(a)



(c)

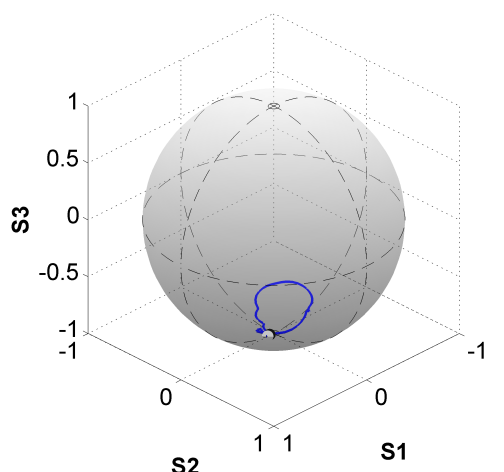

(b)



(d)

Figure 4: Representation of the polarisation transformations on a Poincaré sphere of the transmitted light under the influence of (a) water, (b) acetic acid and (c) ammonia vapours. (d) Temporal change in phase difference induced by each biochemical. White dot: initial state of polarisation; Black dot: final state of polarisation; blue line: path along sphere.

Polarisation response of the silk fibre can be used to detect the presence of vapours with different chemical polarities. Hydrogen bonds within and between molecular protein chains play a crucial role in the silk's structure. Under the influence of these agents, the silk protein bonds are gradually modified, causing the molecular chains to disorient and coil-uncoil. ${ }^{3}$ This effect is then translated into a change in the silk fibre's birefringence, which can be detected by monitoring the state of polarisation (SOP) of the transmitted light. The same experimental setup described in the above section was used to detect several biochemical agents, which impact the hydrogen bonding between the silk proteins within the spider silk fibre. Laser light at $\lambda=1302 \mathrm{~nm}$ was launched inside the silk fibre, the transmitted light was sent to a polarisation state analyser and set to a circular polarisation. A cotton bud soaked with the targeted biochemical agent was then placed close to but not in contact with the 
silk fibre to allow vapour-silk interaction while simultaneously recording the SOP of the light guided through the silk fibre every $0.1 \mathrm{~s}$ for $15 \mathrm{~s}$. Three modifying hydrogen bond-active agents, namely: water, acetic acid and ammonia, were tested and the corresponding Poincaré representations showing the change in SOP in each case is shown in Figure 4(a-c). It is clear from these representations that the chemical agents induced birefringence in the silk fibre.

Table 1: Induced birefringence by each biochemical compound.

\begin{tabular}{|c|c|c|}
\hline Modifying agent & $\Delta B$ & Percentage change (\%) \\
\hline water & $5.11 \mathrm{E}-5$ & 0.270 \\
acetic acid & $4.59 \mathrm{E}-5$ & 0.243 \\
ammonia & $6.38 \mathrm{E}-5$ & 0.337 \\
\hline
\end{tabular}

The temporal evolution of $\phi$ for each experiment was extracted from these measurements and plotted in Figure 4(d). During the first 5-7 seconds of the experiment, the silk fibre was left unperturbed, resulting in no change in $\phi$. When water and acetic acid vapours were placed next to the silk fibre, $\phi$ quickly changed with a response time of the order of the second and went back at a slower rate to its initial phase. In the case of ammonia, $\phi$ did not return to its original value. One plausible explanation could be that ammonia vapours produced an irreversible effect on the orientation of the protein molecular chains. From these values of $\phi$, the change in birefringence $\Delta B$ induced by each biochemical agent was determined and listed in Table 1.

\section{CONCLUSION}

In this communication, the proof of concept of fibre bulk sensing using spider silk has been demonstrated and is illustrated through the biodetection of polar solvent vapours (water, acetic acid, ammonia) by analysing the transmitted light. This result is of significant importance because, firstly, the silk fibre sensor architecture is simple and able to detect polar biochemicals. Secondly, it paves the way for the fabrication of a new generation of economical and custom-made FOBs since spider silk is spun at ambient conditions. Moreover, silks can be functionalized to be even more sensitive to other types of biochemical or particular environmental quantities by incorporating specific dyes making them very cost-effective for potential artificial industrial production of silk-based fibres with unique properties.

\section{ACKNOWLEDGMENTS}

This work was done within the framework of the Ariadna study $\mathrm{n}^{\circ} 14-6401$ (The Silky Way) supported by the European Space Agency. The Oxford Silk group thanks ERC (SP2-GA-2008-233409) and AFOSR (FA9550-121-0294) for funding.

\section{REFERENCES}

[1] Leung, A., Shankar, P. M., and Mutharasan, R., "A review of fiber-optic biosensors," Sensors and Actuators B: Chemical 125(2), 688-703 (2007).

[2] Thévenaz, L., "Next generation of optical fibre sensors: new concepts and perspectives," Proc. SPIE 9157, 9157AN-9157AN-4 (2014).

[3] Shao, Z. and Vollrath, F., "The effect of solvents on the contraction and mechanical properties of spider silk," Polymer 40(7), 1799-1806 (1999).

[4] Huby, N., Vié, V., Renault, A., Beaufils, S., Lefèvre, T., Paquet-Mercier, F., Pézolet, M., and Bêche, B., "Native spider silk as a biological optical fiber," Applied Physics Letters 102(12), 123702 (2013).

[5] Vollrath, F., Porter, D., and Holland, C., "The science of silks," MRS bulletin 38(01), 73-80 (2013).

[6] Little, D. J. and Kane, D. M., "Image contrast immersion method for measuring refractive index applied to spider silks," Opt. Express 19(20), 19182-19189 (2011).

[7] Okamura, Y., Yoshinaka, S., and Yamamoto, S., "Measuring mode propagation losses of integrated optical waveguides: a simple method," Applied Optics 22(23), 3892-3894 (1983).

[8] Kikuchi, K. and Okoshi, T., "Wavelength-sweeping technique for measuring the beat length of linearly birefringent optical fibers," Optics letters 8(2), 122-123 (1983). 